\title{
Decomposing Path Shapes: About an Interplay of Manner of Motion and 'The Path'*
}

\author{
Matthias Weisgerber, \\ Universität Konstanz \\ matthias.weisgerber@uni-konstanz.de
}

\begin{abstract}
In this paper we will develop a formal conceptual model of how the path in a motion situation interacts with the semantic analysis of so called 'motion shape verbs' like 'wackeln' ('wobble'), a subclass of the so called 'manner of motion verbs'. Central to this model will be the distinction between two concepts of motion: translational motion and nontranslational motion, which has no inherent translational component but puts emphasis on describing specific Motion Shape Patterns. We will define and algorithmically describe a theory of Path Shape Decomposition that aims at algorithmically deriving the translational vs. nontranslational distinction from the shape of the path. To account for object internal motion, we additionally introduce Bounding Box encapsulation, which yields a topological division of inner and outer movement. Finally we demonstrate how the outcome of such a technical decomposition can be used in modelling a Path Superimposition scenario like 'Peter wackelt über die Straße'.
\end{abstract}

\section{Introduction}

Compared to path, not much research has been done concerning a formalization of manner of motion. Research in manner of motion has not yet reached a status of formal modelling. It is even unclear what the role of manner information in semantic modelling should be: decompositional semantic approaches do not assign manner an important role in word meaning modelling:

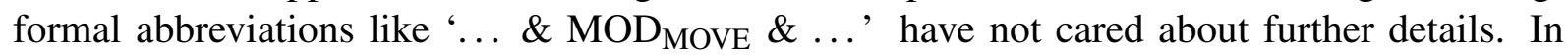
formal semantic representations (e.g. (1), from Kaufmann (1995, p. 225f)), however, the only visible difference in meaning lies hidden in the manner information, which has not been formally elaborated:

$$
\begin{array}{ll}
\text { a. } & \lambda P \lambda x \lambda s\left[\operatorname{GEH}_{P}(x) \& \operatorname{MOVE}_{P}(x) \& P(x)\right](s) \\
\text { b. } & \lambda P \lambda x \lambda s\left[\operatorname{SPRING}_{P}(x) \& \operatorname{MOVE}_{P}(x) \& P(x)\right](s)
\end{array}
$$

The division between the two motion concepts of Go and Move, however, is widely accepted; Talmy $(1983,1985)$ and Jackendoff (1991) elaborate this division. Habel (1999) summarizes this unsymmetry in the state of the art as follows: "Während räumliche Konzepte etwa durch das PATH-Konzept (Jackendoff 1990) - in systematischer Weise in die semantische Beschreibung von Verben der Fortbewegung eingehen, fehlt eine entsprechende systematische Einbeziehung räumlicher Konzepte in der lexikalisch-sematischen Analyse der anderen Bewegungsverben bisher weitgehend. (p. 106) [While spatial concepts like the PATH concepts enter into semantic modelling of motion verbs in a systematical way, there is no systematic theory of

*This research has been supported from the SFB 471 "Variation and Evolution in the Lexicon", funded by the Deutsche Forschungsgemeinschaft. I want to thank Peter Pause, Wilhelm Geuder, Mila Dimitrova-Vulchanova, Liljana Martinez, Joost Zwarts, Matthias Auer and Anja Rüsing for very useful comments and discussions. 
other motion verbs including spatial concepts so far. Translation by author, emphasis added.]". With his analysis of German 'drehen' ('turn'), Habel (1999) presents one first step towards an analysis of the sub-class 'manner of motion verbs'.

Levin (1993, p. 264ff) lists manner of motion verbs in her 'verb classes' collection; and Levin and Rappaport Hovav (1995) further investigate the distinction between Go and MovE as concepts. They define MovE as motion without necessary change of location. Yet what - besides syntactic behaviour, which is a central criterion in Levin (1993) - acts as central feature for this category, what is common to all these verbs? In other words, what makes a verb a 'manner of motion' verb? And, finally, what is the semantic impact of manner of motion?

Maienborn (1994) presents a regularity that explains why sentences like (2-a) are much better than sentences like (2-b): Verbs are able to temporally behave like a translational motion verb and thus subcategorize a path argument.

(2) a. Peter wackelt über die Straße. (Peter is wobbling over the road)

b.???Anja liest in die Küche. (Anja is reading into the kitchen)

A selectional restriction for this effect lies in the connection to contextual and world knowledge: "Das in Frage stehende Prädikat muss auf eine essentielle Eigenschaft der Fortbewegung Bezug nehmen" [The predicate in question has to refer to an essential characteristic of translational motion.] (Maienborn (1994), p. 240). However, Maienborn does not offer a formal model. We will come back to this with a sketch how to apply our model in section 4 .

\subsection{Path Shape Verbs}

Modelling manner of movement can be grouped into at least three components, all containing several conceptual dimensions: ${ }^{1}$ (A) path shape (in which way does the motion relate to the space it is living in); (B) physical parameters of space and time (contact with surfaces, the influence and omnipresence of gravity, speed of motion); (C) an agentive-intensional component, attitudes, and many other parameters (like 'psychological state of figure in motion' - cf. 'gubagguba' in the Language Luganda ('trudge for a long distance with a sad event ahead'), example from Dimitrova-Vulchanova and Martinez (2005)). Consider Dimitrova-Vulchanova and Martinez (2005) for a recent empirical elaboration of dimensions of manner modelling. Based on their classification, one might sketch a Modular Conceptual Space as in (3):

$$
\begin{aligned}
& \left\langle\left\langle\text { РАтн path shape, grain level of specification }{ }^{2}, \ldots\right\rangle,\right. \\
& \langle\text { OвJECT } \pm \text { ANIMATE, } \pm \text { USEOFLIMBS, orientation, intension, attitudes, } \ldots\rangle, \\
& \pm \text { TRANSLATIONAL, } \pm \text { ROTATIONAL, } \pm \text { DEFORMATIONAL, speed, } \ldots\rangle
\end{aligned}
$$

In the current paper we will approach the question how manner of motion information can be described. How can it be anchored to semantics, to conceptual knowledge, to situation representation, and, finally, to the lexicon? And what is the role of the path in this game? We will narrow down the problem onto one of the dimensions: We suggest, while restricting ourselves to an elaboration of Path Shape, that manner of motion verbs express significant micro-variation

\footnotetext{
${ }^{1}$ These dimensions can be modelled as a Modular Conceptual Space, as Geuder and Weisgeber (2006) define it. This offers the advantage that for each module ('domain') the most suitable architecture can be chosen locally. Modules together with intermodule communication establish a Modular Conceptual Space.

${ }^{2}$ As van der Zee and Nikanne (2005) define it: There are three grain levels of Path Shape specification, grain 0: no focus on path shape like in 'go', grain 1: focus on global path shape as in 'curve', grain 2: focus on local path shape as in 'zigzzag'.
} 
on the path involved. We define, in a pretheoretical fashion:

Definition 1 (motion shape verbs) motion shape verbs (class MoM) are those verbs of motion which give more information about details of the motion going on than just starting point, via points and ending point of a path. They need not be specified for a change of place.

Note that this class is orthogonal to what is often called verbs of locomotion (see, for example, Eschenbach et al (2000)); and in our case it is definitely not meant to be a basis for categorization - since we assume scales of increasing informativeness of manner representations, as in 〈'go' $<^{*}$ 'fahren' ('drive'), 'fliegen' ('fly') $<^{*}$ 'wackeln' ('wobble') $\rangle$, where $<^{*}$ is a suitable measure.

Examples for motion shape verbs are: 'crawl', 'creep', 'wobble', 'shiver', and many others. A subclass is the class of pure Path Shape Verbs like 'spiral', 'curve', 'zigzag'.

Consider, as an example, (4): ${ }^{3}$

30 Tonnen Waren wackeln auf den Köpfen von rund 650 Lastenträgern auf 30 tons of goods are wobbling on the heads of about 650 carriers on Bergpfaden in Richtung Marktplatz. mountain paths towards market place.

(http://www.spiegel.de/wissenschaft/mensch/0, 1518, 360820,00.html, 17.6.2005)

The theme of the motion situation given in (1) ('30 Tonnen Waren') is being transported along an atelic (unbounded) path with specified Via ('auf Bergpfaden') and Direction ('in Richtung Marktplatz'). The verb 'wackeln', however, does not basically express translational movement but a movement shape: while fixed at a position, the theme moves in a defined cyclic pattern with a defined speed.

Finally, how should meanings of verbs like 'wackeln' and the combination with a path-PP be lexicalized? In the course of this paper, we will argue that a path can be divided into cyclic patterns and a translational component and that linguistically, the translational components refer to (intended) motion from a source $a$ to a goal $b$ as expressed in PPs, while the cyclic patterns refer to manner-of-movement information as expressed in path shape verbs and -adverbs. In the following sections we will first see which hints and answers current research is offering, we will then analyse the connection between motion and path shape. In a next step we will formally introduce Path Shape Decomposition, starting from a discussion of technical requirements. We will demonstrate that the shape of a path is the result of merging a translational source-goal component (e.g. as expressed in the path-PP) and a number of what we call movement shape patterns (normally implicitly expressed in manner-verbs or -adverbs). Finally we will discuss some case studies and provide examples for lexical entries. 4

\subsection{Decomposing Motion}

Engelberg (2000) argues in favour of an analysis assuming two parallel subevents, and presents linguistic as well as psychological evidence. He calls manner of motion verbs Zweibewe-

\footnotetext{
${ }^{3}$ We have tried to give English translations for all German examples. These glosses, however, do not in all cases provide a 1:1 mapping of sense. Also, judgements of examples cannot be directly transferred here.

${ }^{4}$ Note, additionally, that our notion of Path Shape is different from, but not contradictory to, what Zwarts (2006) calls 'event Shape': his proposal is to include a Path notion into the lexical meaning of verbs like 'enter' and prepositions like 'into' such that the pairwise similarity between both in spatial terms is reflected in a parallel construction of the lexical entry. Doing so, however, he remains on a grain level which does not affect what we call 'motion shape'.
} 
gungsverben $(\approx \text { dual movement verbs })^{5}$ : he assumes two movements taking place simultaneously in the same event: a translational movement and, relative to that, an eigenmovement of the participant. He assumes these two subevents as central components of the semantic structure. Put in Path Shape analytical terms: there is a relation between translational motion along a path on the one hand and cyclic motion patterns performed by the object on the other. Path Shape Decomposition can here be taken as a formal account to more formally describe this interplay by linking the path shape patterns to subevent descriptions in order to see which is the influence of both subevents onto the resulting Path Shape.

Shaw, Flascher and Mace (1994, p. 485f) report the observation that subjects decompose observed motion. The motion of a rolling wheel is recognized as a decomposition of a translation of the middle point and a rotation of another point round the middle point. Therefore the authors claim that decomposition of the event leads to a more basic way of describing a complex motion event. This finding backs our approach, since we believe that path decomposition enables us not only to describe and represent motion events as a whole, but also that most basic patterns of a complex motion are conceptually linked to the meaning of manner of motion verbs.

Musto et al (2000) report the empirical finding that when subjects observe moving dots on a screen and after it draw the path how they remember it, performance increases (or even overgeneralizes) when subjects recognize certain patterns in the path. This, again, supports our argument that decomposing the Path is an efficient way of analysing the informational content of Path motion situations.

To conlude: A translational and a cyclic nontranslational motion component can be present within the same verb. This results in a complex path shape: Whenever in a motion event the path is significantly not neutral (grain 0 ), the path shape can be decomposed into a sum of more simple Path Shape Patterns which are linked to the meaning of manner verbs and -adverbs. In the following we will finally present the Path Shape Decomposition framework. We will see how a Path Shape decomposition is used to form the link to lexical modelling of motion shape verbs.

\section{Path Shape Decomposition}

In this chapter we will develop a formal conceptual model of how the path in a motion situation interacts with the semantic analysis of motion shape verbs. Central to this model will be the distinction between two concepts of motion. - The first is a concept of translational motion. This component can be modelled by a suitable path theory, as has been proposed in various approaches in literature, and as we are also modelling in other current work (Weisgerber forthcoming). The second motion concept has no inherent translational component but puts emphasis on describing specific motion patterns. The latter cannot be described by current path theories: semantic path theories are not designed to represent path in a granularity that is both fine enough to represent a motion in all its details, and technically equipped to account for cyclic path shapes that emerge from this motion.

In order to account for this problem we will decide on a pointwise path definition that allows for a fine grained focus. We will define and algorithmically describe a theory of Path Shape Decomposition that aims at algorithmically deriving the translational vs. nontranslational distinction from the shape of the path.

\footnotetext{
${ }^{5}$ all terms originally German, English terms suggested by the author of this paper
} 


\subsection{Introducing the model}

To start with we define the distinction between translational and nontranslational movement:

Definition 2 (translational vs. nontranslational movement) Let, preformally, a place be a 'possible location for an object in space'. We call a movement a translational movement if it is a movement of an object on a path starting at a source and ending at a goal ( $\rightarrow$ 'change of location') and no place is visited more than once. We call a movement a nontranslational movement if it is a movement pattern with no source and goal defined, where the object repeatedly returns to a place or a position after a short finite time. ${ }^{6}$

Take, for example, 'go' as a translational movement: an object moves on a path from a source $a$ to a goal $b$; and take 'wobble' as an example for a nontranslational movement: an object starts moving at a position $a$ and passes by this position regularly after some finite time. Many verbs, however, express both components (e. g. 'jump', 'walk'), and some verbs are able to change between expressing translational or nontranslational movement depending on the context and the reference system (e.g. 'turn' is , by the definitoin given, undecided between being translational or nontranslational). Therefore, this distinction of translational vs. nontranslational is no basis for different verb categories. Consider, as an example, sentence (5):

Der Käse rollte zum Bahnhof. (The cheese rolled to the station)

This 'roll'-situation includes two kinds of movement: first, there is a circular rotation pattern - an object rotates with contact to the ground (the core meaning of 'rollen') -, and second, there is a translational movement, which is introduced by the goal-PP. Since both motions are linear within time, they can be added up, yielding a sine shaped path for every point of the moving object.

Central for our analysis is the following fact, that obviously follows from both geometry and functional analysis:

Fact 1 (Path Shape Decomposability) Every sequence of subsequent positions can be decomposed into a finite number of cyclic patterns and an optional translational component. ${ }^{7}$

Linguistically, the translational components refer to (intended) motion from a source $a$ to a goal $b$ and the cyclic patterns refer to manner of movement information. In Satellite-framed languages ${ }^{8}$ the first is 'normally' expressed in PPs, while the second is 'normally' expressed in manner of motion verbs and -adverbs - however, this linking can be realized in various variants.

\footnotetext{
${ }^{6}$ The expression 'after a short finite time' reminds of the unavoidable pragmatical influence of the notion of space and time in the reference system, which can be seen in the unprototypicality of the use of 'wobble' in 'imagine a planet that wobbles between two suns with a frequency of some 100.000 years'.

${ }^{7}$ The mapping between rotations as circles and their representation as sinus functions is a common mathematical notion. That means, a complex motion shape (in rotation interpretation) can be converted into a complex sine function. Using Fourier Analysis, this can be decomposed into basic sine functions with amplitude and frequency, which corresponds to radius and rotation speed of a circle

Note in this context that 'cyclic patterns' is not specified for another aspect of shape yet: both the abrupt change of direction in 'zigzag' and the more rotational shape in 'swing' or 'circle' is subsumed here. Fourier analysis, on the other hand, can extract a sine in one single step, whereas a zigzag yields infinite combination of sine functions. This may be taken seriously as a hint that from a physical point o view zigzagging is not a natural basic object motion pattern. Indeed, zigzagging in real world tends to be eihter round-edged or an alternating sequence of straight-line motions intervals and turn-on-position motions, hence it is, physically, not one basic motion pattern. However, consider Zee (2000) for an investigation of the sharp edge feature in zigzagging.

${ }^{8}$ following the Talmy-classification, although this classification raises some unanswered questions.
} 
Path Shape Decomposability and the fact that motion pattern information is expressed by words, i. e. is part of their lexical meaning, implies that there are two possible directions of mapping to be modelled: they can be subsumed as linguistic analysis and linguistic generation (cf. fig. 1). The lingusitic analysis direction is a mapping of linguistic motion situation descriptions to a

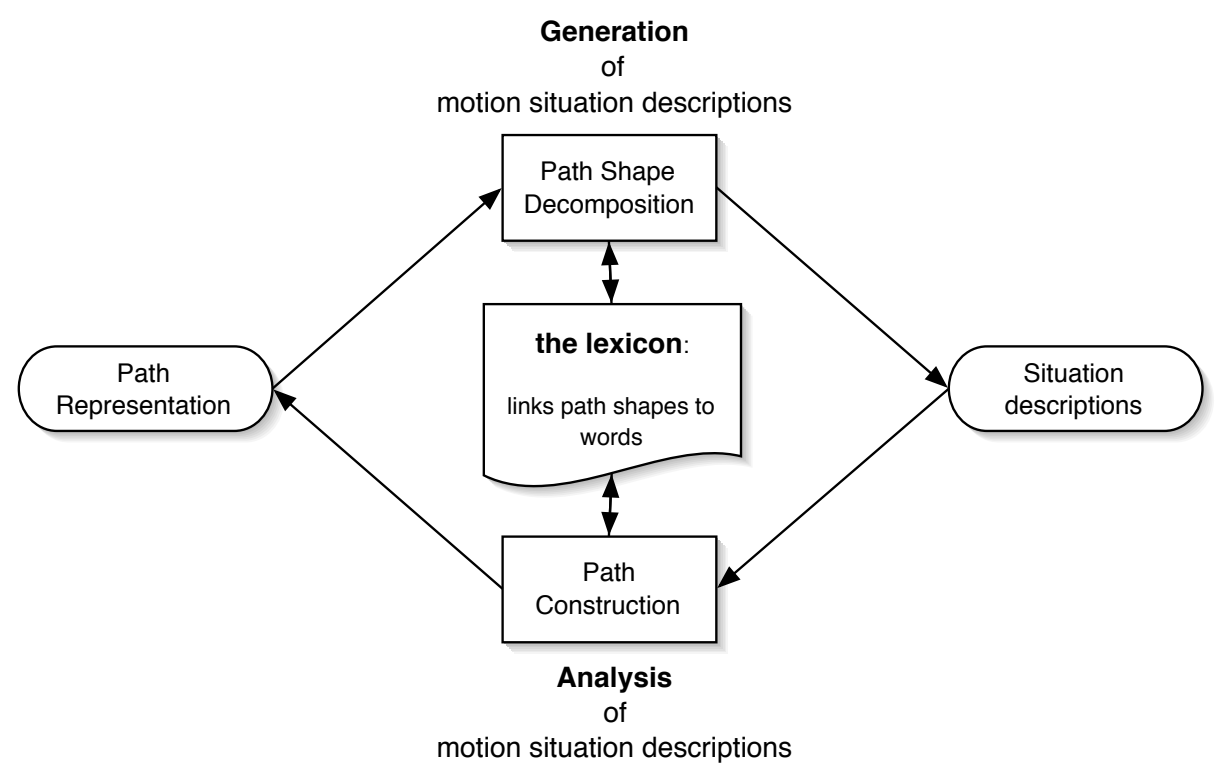

Figure 1: Two directions of PSD

model representation of the path and manner patterns involved. This direction requires a lexicon which links path shape building blocks to words and a theory that allows mounting these parts together to yield a path shape representing the situation. For a given sentence like 'Peter wobbles from $a$ to $b$ ', an algorithm will produce a path-geometric analysis of the situation described. The linguistic generation direction, on the other hand, describes the reverse process: it is a mapping of a physically given path representation to language. Given a formal graphical description of the path shape, the algorithm generates a sentence that describes the situation as linguistic output, using both path and manner expressions. The latter direction is both algorithmically and linguistically of high complexity: the algorithmical part consists of decomposing the path in parts which are each linked to words in the lexicon, and the linguistic part generates natural language output. Due to finiteness of space in the current paper we will not discuss the latter part here.

\subsection{Defining the toolkit}

Technically, the model we suggest operates on a simulation level of situation representation, called $\sigma$ level, whose task it is to build physical models of the situation, according to the knowledge provided by semantic and conceptual levels, and to judge the physical (im)possibility of a situation described in the actual world settings. This level can be seen as the interface between conceptual and world knowledge about physical space.

Similar to Zwarts (2004b), who suggests a path algebra defining path as "a starting point, an end point, and points inbetween on which the path imposes an ordering [...] defined as continuous functions from the real unit interval $[0,1]$ to positions in some model of space", we define the path as a sequence of location-relations between a moving theme and a background object. ${ }^{9}$

\footnotetext{
${ }^{9}$ This definition offers the advantage that inserting and deleting path points - as is done when increasing and
} 
Definition 3 ( $\sigma$-Path) A Path in the $\sigma$-world is a chain of points, two of which are designated as starting point and end point:

$$
\begin{aligned}
& \text { PATH }=\left\{x_{i} \in \operatorname{PoS}, i \in[0 . .1] \subset \boldsymbol{Q}:\right. \\
& \quad \operatorname{NeIghbour}\left(x_{i}, x_{j}\right) \& \operatorname{NeIGHBour}\left(x_{j}, x_{k}\right) \text { iff } \\
& \left.i<j<k \wedge \neg \exists x, y: i<x<j<y<k \wedge x_{0}=\text { 'starting point' } \wedge x_{1}=\text { 'end point' }\right\} .
\end{aligned}
$$

\subsection{The Lexical Entries}

In the path generation algorithm, which starts out from linguistic input and ends with printing out a path shape, this connection is algorithmically represented as a step 'link word meaning to path representation $\leftarrow$ use $\leftarrow$ lexicon'; and in the Path Shape Decomposition algorithm it would be the step 'linguistic generation [from path shape snippets] $\leftarrow$ use $\leftarrow$ lexicon'. That assigns a key role to lexical entries: they are the central data structure that bidirectionally links path shape to language. Let us shortly give two examples: German 'wackeln' ('wobble') and 'to spiral'.

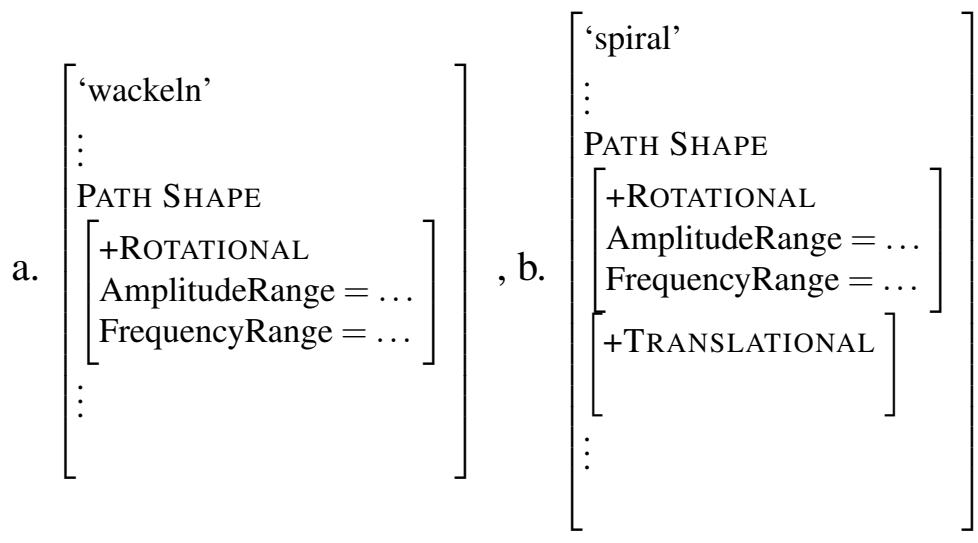

The excerpt from a lexical entry for the item 'wackeln' shows the link between Path Shape Snippet and Lexicon. The Path Shape that belongs to the (spatial) meaning of 'wackeln' can be defined in terms of a range of possible Amplitude values and a range of possible frequency values, which together yields a sine shaped Path snippet. Furthermore 'wackeln' is purely +Rotational, that means it is not translational and hence does not offer a slot for a PP as an argument. This yields path superimposition.

The verb 'to spiral', a Path Shape verb, is an interesting case, since it is the 'prototype' for a combination of a translational and a rotational component. Note that there are many ways to compose the translational with the rotational component: it depends on the angle between the plane of the rotation and the direction of the translation - hence, the verb is underspecified for this distinction: all constellations are good evidences of 'spiral'. If the translation is orthogonal to the plane of the circular component, we get a 'cylindrical' spiral (as in 'spiralling up around the pilar'), and if they are in the same plane, we either get a standard spiral (as in 'spiralling towards the sun') or a translation where the object is performing circles. Consider Zee (2000) and Zwarts (2004a) for an in-depth analysis of 'to spiral'.

decreasing granularity, respectively - only means rewriting two neighbour pairs, which is of little algorithmic complexity. Additionally, one can assume replacement functions 'starting point $\rightarrow$ source' and 'end point $\rightarrow$ goal' dependent on the decision if the path is telic (as in 'arrive') or atelic (as in 'approach'). We do not elaborate on that - see, for instance, Zwarts (2004b), Verkuyl (1993) and Verkuyl and Zwarts (1992) for an elaboration of aspect and (a)telic path. 


\subsection{The Algorithm}

Having defined a toolbox and having defined the structure of the lexical entries that we assume, we are finally ready to dive into the center of the path shape decomposition approach: the algorithm.

The Path Generation Algorithm is given in figure 2. The input is a linguistic representation of a motion scenario. This representation is linguistically decomposed by standard syntactic and semantic tools. In this process all word meanings are looked up in the lexicon, which contains path representation patterns for motion vocabulary. Words are linked to path representation patterns. These patterns to path are linked to the path, which is gradually built up stepwise. The whole process is called recursively, along the recursive structure of the linguistic decomposition tree. The recursion ends when the whole sentence is analysed and at the same time the whole path is built. The output is the path shape that belongs to the sentence which has been put in.

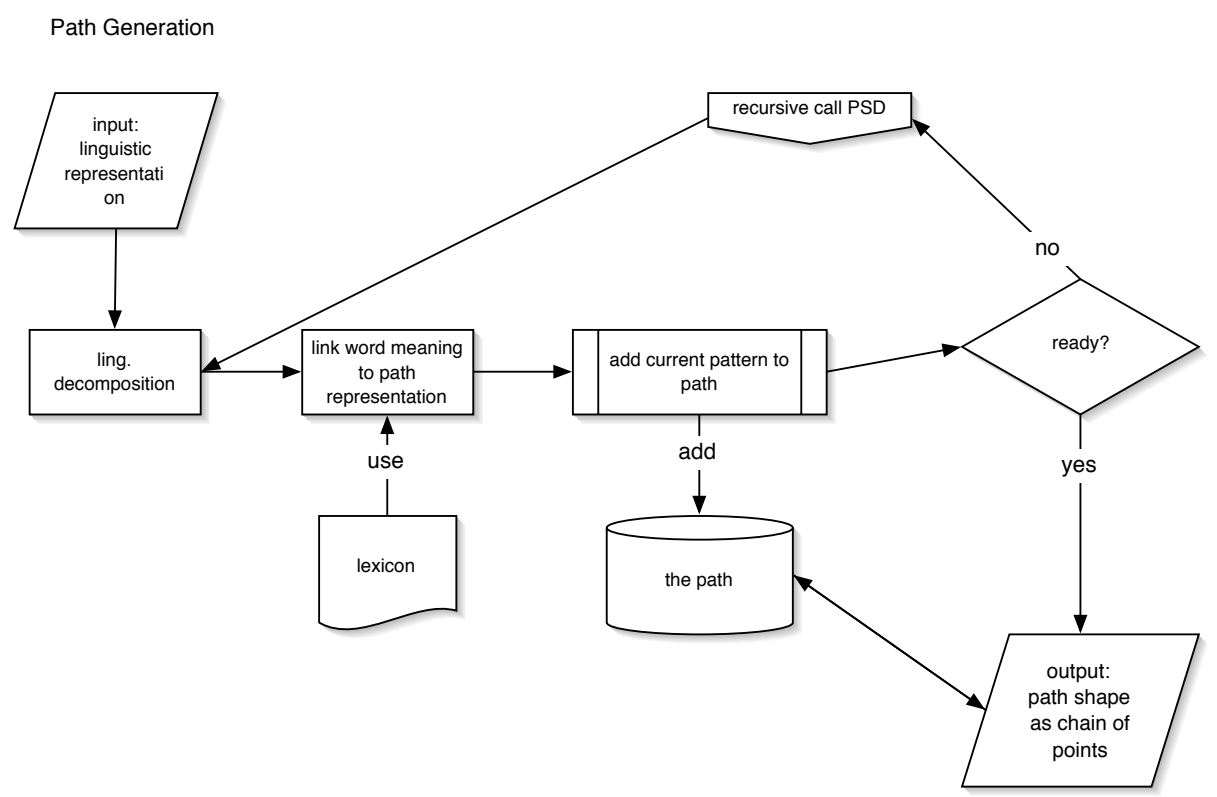

Figure 2: Path Generation Algorithm

\subsection{Conclusion}

This section has been the 'inventive' part of the paper. We have argued that in motion situations the path can always be decomposed in a number of rotation patterns and one optional translation (Path Shape Decomposability). We have defined, as a toolkit, the $\sigma$-world and a notion of path as chain of points. Finally, we have proposed the Path Generation Algorithm, which models the Linguistic Analysis direction. The duty of the Applications section 4 will then be to make clear how this information is dealt with in concrete by the algorithms. Before, however, we have to address a class of cases that has not been addressed so long: motion situations including rotation and deformation, as well as other cases of object internal motion. 


\section{Higher dimensional object representation: the Bounding Box.}

While in a majority of situations involving path the relevant positions of the whole object can be modelled as points (which directly fits into the pointwise definition of path, as in 'Peter went to Trondheim'), things are different in cases where deformation or rotation are involved (as in 'Maria bent forward', 'Peter turned round'). In these cases, the object does not only move as a whole along a path, but subject to its physical architecture, it undergoes shape changes and orientation changes. Furthermore, a 'translational' and a manner component can be present within the same verb. It need not be the case that both components are fully lexically specified - consider 'springen', 'hüpfen' ('jump'), cf. discussion on pairs in Dimitrova-Vulchanova and Weisgerber (in process).

\subsection{Rotation.}
a. The record is turning.
b. ??The record is turning towards the door.
c. ??Peter is turning.
d. Peter is turning towards the door.

Although each point of the object moves on a path in the course of the rotation, the object as a whole does not change position. Even if we consider a real translational movement of the single points of the record, the reading as change of position remains semantically bad (as in (7-b)) this is due to the fact that there is no outstanding point which gives the record an implicit main axis.

Another problem mentioned above becomes visible in (7-d): 'turn' either is purely rotational or it is both tranlational androtational, i.e. there is an optional translation involved in the meaning of turn. We will come back to this case (and for an in-depth elaboration of rotation consider Habel (1999).)

\subsection{Deformation.}
a. Maria bent out of the window.
b. Maria bent to front.

In (8-a) two aspects interfere: one part of Maria changes its position moving on a path from inside through the window to outside; another part of Maria, however, does not change position: even if most parts of Maria are outside, we still recognize Maria as inside the window. We claim this effect is both a matter of the focus we put on the different body parts - as long as Marias feet are inside and Maria is standing on her feet, the position 'inside' is assigned to the whole of Maria - and a matter of which chain of changes of positions lead to the actual position - all of Maria was inside before the movement, and she will end up inside again after the bending process. The same effect remains more implicit in (8-b), where a part of Maria moves to a front position while Marias overall position in space remains unchanged. Finally, there is additional semantic evidence for an analysis where (8-a) does not describe a change of place: The bending situations behave like states, and changes of state can be added separately - consider (9).

(9) a. Maria bent out of the window for three hours / * in three hours.

b. *Maria bent out of the window and back again.

c. Maria bent out of the window, and then she fell out of the window. 
This fact will strengthen our analysis that the location of the motion event is, with no change, inside the room - although parts of the object (Mary) are located outside the window.

\subsection{Internal vs. External: Encapsulation in the Bounding Box}

These cases of 'object internal' motion lead to a granularity where we have to treat the dimensionality of the object as greater than zero (i. e. 'point'). In the case of deformation, single points of the object are able to perform motion relative to the whole - this object-internal motion depends on the physical character of the object. The increase in the dimensionality of the object influences the modelling of the interplay of object and path: A topological division of movement inside the object and movement outside the object arises. This division is a key to ambiguity effects arising from the fact that it is not always clear where, relative to the object, a movement is located: it is, therefore, not obvious which is the reference frame of a movement.

All of this implies that the model has to account for such cases - in other words, the model needs an object representation tool. Can this be formalized without the cost of unbearably high complexity? Let us answer this question in two steps: At first, we show that an additional modelling of object internal movement is possible with finite effort. This is due to the following:

Fact 2 If an object changes its shape (internal deformation) without infinitely increasing its volume, then the process of extension is a finite process in all dimensions: in the extreme case, all available volume extends along one single dimension - the object has changed into approximately a line of finite length, and cannot extend any more. Hence, if the possible deformation is finite on all dimensions, all possible deformations can be described as patterns, i. e. the process stops after some time or returns to a known former state.

The second step is that we distinguish between 'inside' and 'outside' of objects: We assume a Bounding Box as a model of the object in the $\sigma$-world. The Bounding Box 'wraps' (encapsulates) the entire object and thus clearly defines a border between inside and outside:

Definition 4 (Object Encapsulation: Bounding Box) A Bounding Box BB of an object $O$ in the $\sigma$-world is a cohesive cover of points, which encloses the object $O$ :

$\mathrm{BB}(O):=\left\{x_{i, j} \in \mathrm{PoS}, i, j \in[0 . .1]:\right.$

(i) $x_{i, j}$ represents a point of the object and has a neighbour that does not belong to the object or (ii) there is a plane through $x_{i, j}$ such that more than 2 neighbours of

$x_{i, j}$ lying on that plane represent object points.

This set is constructed recursively. A model of an object $O$ in the $\sigma$-world involves exactly one active Bounding Box $B B(O)$ in each context and point of time. This Bounding Box divides the inside from the outside. ${ }^{10}$

\footnotetext{
${ }^{10}$ Encapsulating the object in a Bounding Box is our model's way to deal with cases that involve vagueness. Vagueness can appear in several cases - we would like to mention only the cases of object shape vagueness (in a class of objects it is difficult to define which is the exact extension of the object, e. g., where exactly does a cloud end?) and region vagueness (e.g., 'flowers in the vase' or 'apples in the bowl', cf. the seminal work about language and cognition of spatial prepositions by Herskovits (1986)). It is central to our notion of Bounding Box that we will put vagueness into bounds rather than analyse it away or eliminate it: At a given point of time, the Bounding Box does not equal but approximate the size of the object, and thus stands for the object to allow further reasoning with the situation. That means that vagueness is shifted to the process of assigning the Bounding Box: the more vague the object shape, the more context, pragmatics and reasoning enters into the process of Bounding Box assignment. ${ }^{11}$
} 
We now apply the topological division of inside and outside to the relation between object and movement:

Definition 5 (Object Internal vs. -External Movement) The division of movement into translational and nontranslational movement is applicable in a recursive way: the Bounding Box representing the object makes up a reference system, in which translational and nontranslational movement can take place again. Movement inside the Bounding Box is called object internal movement, while the movement of the Bounding Box relative to a larger reference system is called external movement.

Note that due to the recursivity the notion of Bounding Box induces a reference system with inside and outside in all cases. Take, for example 'The stain on the record moves to the left'. The Bounding Box of 'the stain' is now in focus, it is moving on a path that itself is located inside the Bounding Box of the record. That means that, relative to the record, there is no movement of the stain (it is fixed to the surface), relative to the outside world, the movement of the record is internal (the record remains fixed at its place as whole) and the motion of the stain is external (it is being transported along a path). Since this division is triggered by the Bounding Box, which is set dynamically due to both the verb's lexical entry and influences of the context, it becomes clear once more that this division cannot be a basis for a stable verb categorization (as we have discussed above).

It is common to all physical objects in real world that they are located at one place due to environment forces. Gravity, which creates contact between an object and the ground, can be argued to be the instantiation of 'support'. This physical fact directly enters into our Bounding Box framework: There is a subset of Bounding Box points that are involved in contact to another supporting object due to environmental forces. We call this set of points the fixation plane of the Bounding Box. The fixation plane anchors the Bounding Box to the space it is "living" in. Note that the fixation plane needs not be flat - its shape is influenced by the shape of the supporting ground.

Let us now go back to two examples of situations, repeated here as (10-a) and (10-b), and see what effects can occur within the Bounding Box framework.

a. Maria bent out of the window.

b. Peter turned to the left.

As we have argued above, (10-a) does not describe a change of place but an internal movement. No external movement of an object on a path is taking place here. This is modelled with the help of the Bounding Box of the object 'Maria': While Maria is moving parts of her body out of the window, the Bounding Box representing a model of the object Maria has to extend to cover the whole object. The place of the Bounding Box, its position in space, remains unchanged, since the fixation plane is stable: Maria is standing on her feet. The fact that bending is object internal, finally, is a feature of the verb 'bend': in the lexical entry of 'bend' the feature +INTERNAL must be present, and no path slot. Example (10-b) concerns change of orientation. The shape of the object Peter evokes an internal orientation axis: Peter has a 'front' and a 'back'. The verb 'turn' has the meaning of a change of the absolute direction of this orientation. This makes the example ambiguous in that without context we cannot infer if the turning is internal (turn on a point) or external (move on a circled path). This ambiguity is a regular one - it has to be fixed in the verb entry as \pm EXTERNAL. In the external case the fixation plane of the Bounding Box of the object 'Peter' moves on a circled path, whereas in the internal case, the fixation plane remains at a fixed position and the movement takes place inside the Bounding Box. What about the PP? In both cases, external and internal, a path PP can be present ('turn into Tägermoos 
road' vs. 'turn to the left'). Syntactically that means that the verb allows for a PP in each case, and the lexical entry of the verb has to decide on the meaning of the PP: in the external case, the PP is linked to a (circled) path, whereas in the internal case, the PP is linked to the direction of the orientation vector.

Finally, of what help can a bounding box be in the path superimposition case? Consider

Peter zittert über die Straße (Peter is shivering over the road).

Again, the Bounding Box defines a reference system. When talking about situations, one cannot switch reference system, therefore it is impossible to mention inside- and outside-information together in the same clause. ${ }^{12}$ So, how does inner information get to outside? An enfocusstrategy makes the Bounding Box more narrow (i.e. change the referency system) as to make inner motion visible to the outside as motion of the whole Bounding Box. Consider 'zittert über die Straße' (to be discussed later): If the shivering affects outer path shape, then it has become a shivering of the whole Bounding Box. The motion of the Bounding Box is what is superimposed in the end. ${ }^{13}$

\section{Degree of influence, Maienborn's 'temporary motion verbs', and $\lambda P$}

Maienborn (1994) deals with cases where verbs that lexically do not provide a path slot are combined with path-PPs. Consider the following examples (taken from Maienborn) - all of these verbs are no change of location verbs; and only some of them are (manner of) motion verbs.

(12) a. Ein Motorrad knattert über die Landstraße. (A motorbike crackles over the road)

b. Der Hochgeschwindigkeitszug dröhnt durch den Tunnel. (The high speed train booms/drones through the tunnel)

c. Das Motorrad jault durch die Stadt. (The motorbike whines through the city)

d. Gunda turnt über den Sessel. (Gunda does-gymnastics over the armchair)

e. Gunda hampelt in die Küche. (Gunda (actively wobble around) into the kitchen)

f. Das Kleinkind wackelt in die Sandkuhle. (The small child wobbles into the sandbox)

(13) *Gunda liest in die Küche. (Gunda is reading into the kitchen)

How does, semantically, the path anchor to the meaning of the verb? Maienborn (1994) argues against a notion of pure modification and proposes instead a mechanism where the verb becomes a temporary motion verb. This meachanism is triggered by the path-PP.

As can be seen in (13), however, this mechanism needs to be restricted: "Das in Frage stehende Prädikat muss auf eine essentielle Eigenschaft der Fortbewegung Bezug nehmen" [The predicate in question has to refer to an essential characteristic of translational motion.] (Maienborn (1994), p. 240).

In the case of manner of motion verbs, path shape analysis can be of some help: If a verb encodes information about any kind of motion and if this motion is not purely internal but has a visible effect onto the resulting path, it is possible to semantically superimpose this motion

\footnotetext{
${ }^{12} \mathrm{cf}$. Bohnemeyer (2003) for an empirical crosslinguistic investigation how many motion path information can be encoded in one clause

${ }^{13}$ However, this is not completely trivial (see also discussion on 'wackeln'). Which point of the object defines the path that I recognise as 'zigzag'? Imagine the objects is fixed to the carrier and therefore only wobbles with its upper end. We define: the greatest existent amplitude is taken as the amplitude of the pattern motion.
} 
on a path introduced by a PP, and reversely, to treat the PP temporarily as an argument of the situation representation. Compare:

(14) a. Peter wackelte über die Straße. (Peter was wobbling over the road)

b. ?Peter zitterte über die Straße. (Peter was shaking over the road)

c.???Peter fror über die Straße. (Peter be-cold- $V_{\text {fin }}$ over the road)

Interpretation: The movement induced by the manner pattern must have an influence on the translation movement: 'wobbling' and 'over the road' must interact. ${ }^{14}$ (14-b) is another evidence for that: There is one possible reading of (14-b) where the effect of shaking is visible in Peters movement. The more of the pattern motion effect is visible, the better the sentence. Hence, the amplitude of the pattern-motion is significant for meaning distinction: 〈'wackeln' > 'schwingen' $>$ 'zittern' $>$ 'vibrieren' $\rangle$.

In the case of sound emission verbs, one has to 'dive deeper into context' - but, in the end, the same claim holds, when we assume the causation relation: the motion on the path produces the sound emission, a 'trace of sound' can be recognized for a while. But this has to be elaborated in depth at another place.

Finally, consider once more (4) repeated as (15) - which seems to contradict Maienborns thesis that a verb can provide a $\lambda P$ slot whenever it wants to:

30 Tonnen Waren wackeln auf den Köpfen von rund 650 Lastenträgern auf

30 tons of goods are wobbling on the heads of about 650 carriers on

Bergpfaden in Richtung Marktplatz.

mountain paths towards market place.

Here, the combination of the rotational pattern part and a Path-PP cannot yield translational reading ((16).c is out as an interpretation of (15)). That should be taken as a sign for the nonexistence of a $\lambda P$ slot in the verb. Path Shape Superimposition is the only remaining possible interpretation: the pattern motion is superimposed on a path, hence it is not itself the path.

a.
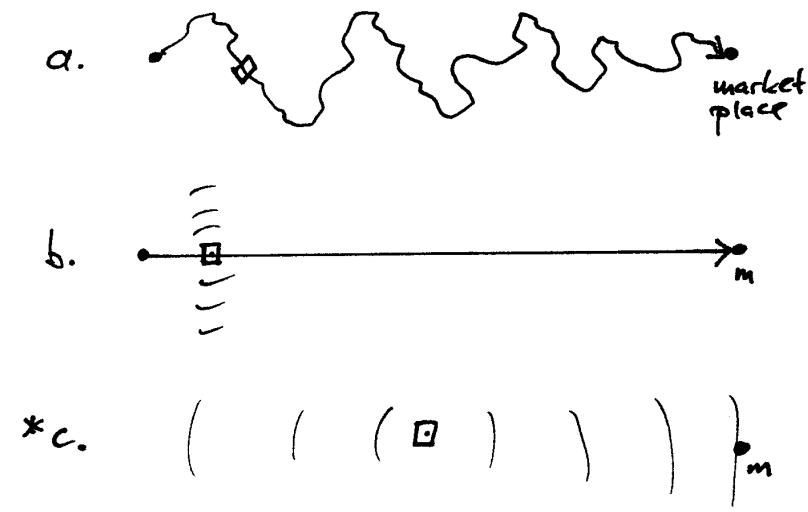

\footnotetext{
${ }^{14}$ This effect has been called Path Superimposition. "Superimposition is a graphics term meaning the placement of an image on top of an already-existing image, usually to add to the overall image effect, but also sometimes to conceal something (such as when a different face is superimposed over the original face in a photograph). [en.wikipedia.org/wiki/Superimposition]".
} 


\section{Conclusion: Path Shape Decomposition and Manner Modelling}

(Modified) manner of motion verbs yield one single complex path of motion. With the help of the Path Shape Decomposition framework we presented, this path can be seen as consisting of two kinds of components: iterated rotational patterns and one translational part. These are linked to the lexical meaning of manner verbs and -adverbs: Motion Shape Patterns are in most cases linked to 'manner' information, while the translational component is often expressed by the Path-PP or direction adverbs. In order to account for object internal motion, we additionally introduced Bounding Box Encapsulation, which yields a topological division of inside and putside-movement. As an application, we discussed the 'wobble over the road'-case and related cases and presented an explanation for Maienborns path-superimposition effect .

We are aware of the fact that many details have to been added to the framework. Many facets are in preparation, and others are considered to be projects of 'further work'.

\section{References}

Bohnemeyer, J.: 2003, The unique vector constraint: The impact of direction changes on the linguistic segmentation of motion events, in E. van der Zee and J. Slack (eds), Representing Direction in Language and Space, Oxford University Press, Oxford, pp. 86-110.

Dimitrova-Vulchanova, M. and Martinez, L.: 2005, Motion naming in three satellite-framed languages: A pilot study. Paper given at Scandinavian Conference on Linguistics (SCL) 21, Trondheim, 2.6.2005, unpublished.

Dimitrova-Vulchanova, M. and Weisgerber, M.: in process, Pairs, unpublished, NTNU Trondheim \& Universität Konstanz.

Engelberg, S.: 2000, Verben, Ereignisse und das Lexikon, Niemeyer, Tübingen.

Eschenbach, C., Tschander, L., Habel, C. and Kulik, L.: 2000, Lexical specifications of paths, in Freksa, Brauer, Habel and Wender (2000), pp. 127-144.

Freksa, C., Brauer, W., Habel, C. and Wender, K. F. (eds): 2000, Spatial Cognition II, Integrating Abstract Theories, Empirical Studies, Formal Methods, and Practical Applications, Vol. 1849 of Lecture Notes in Computer Science, Springer, Berlin.

Geuder, W. and Weisgeber, M.: 2006, On the Geometrical Representation of Concepts. Reflections on Peter Gärdenfors (2000): Conceptual Spaces, under revision for: Linguistics and Philosohpy pp. ??-??

Habel, C.: 1999, Drehsinn und Reorientierung - Modus und Richtung beim Bewegungsverb drehen, in G. Rickheit (ed.), Richtungen im Raum, Westdeutscher Verlag, Opladen, pp. 101-128.

Herskovits, A.: 1986, Language and spatial cognition. An interdisciplinary study of the prepositions in English, Cambridge University Press, Cambridge, Mass.

Jackendoff, R.: 1991, Semantic structures, number 18 in Current studies in linguistics series, MIT Press, Cambridge, Mass.

Kaufmann, I.: 1995, Konzeptuelle Grundlagen semantischer Dekompositionsstrukturen. Die Kombinatorik lokaler Verben und prädikativer Komplemente, Niemeyer, Tübingen. 
Levin, B.: 1993, English Verb Classes and Alternations. A Preliminary Investigation, The University of Chicago Press, Chicago / London.

Levin, B. and Rappaport Hovav, M.: 1995, Unaccusativity. At the Syntax-Lexical Semantics Interface, MIT Press, Cambridge, MA.

Maienborn, C.: 1994, Kompakte Strukturen - Direktionale PPn und nicht-lokale Verben, in S. W. Felix, C. Habel and G. Rickheit (eds), Kognitive Linguistik. Repräsentation und Prozesse, Westdeutscher Verlag, Opladen, pp. 229-249.

Musto, A., Stein, K., Eisenkolb, A., Röfer, T., Brauer, W. and Schill, K.: 2000, From motion observation to qualitative motion representation, in Freksa et al. (2000), pp. 115-126.

Shaw, R. E., Flascher, O. M. and Mace, W. M.: 1994, Dimensionen der Ereiniswahrnehmung, in H. Feger and J. Bredenkamp (eds), Wahrnehmung (Enzykolpädie der Psychologie. Themenbereich C: Theorie und Forschung. Serie II: Kognition. Bd. 1), Verlag für Psychologie Dr. C. J. Hogrefe, Göttingen, pp. 457-528.

Talmy, L.: 1983, How language structures space, Spatial Orientation: Theory, Research and Application, Plenum, New York, pp. 225-282.

Talmy, L.: 1985, Lexicalization patterns: semantic structure in lexical forms, in T. Shopen (ed.), Language typology and syntactic description, Vol. 3: Grammatical categories and the lexicon, Cambridge University Press, Cambridge, MA.

van der Zee, E. and Nikanne, U.: 2005, The grain levels in the linguistic expressions of motion. Talk given at Scandinavian Conference on Linguistics (SCL) 21, Trondheim, 2.6.2005.

Verkuyl, H.: 1993, A Theory of Aspectuality, Cambridge University Press, Cambridge / Mass.

Verkuyl, H. and Zwarts, J.: 1992, Time and space in conceptual and logical semantics: The notion of path, Linguistics 30, 483-511.

Weisgerber, M.: forthcoming, Where lexical semantics meets physics: Towards a three-level framework for modelling path, in M. Dimitrova-Vulchanov and E. van der Zee (eds), - to appear, title to be announced -, Oxford University Press, Oxford, p. ?

Zee, E. v. d.: 2000, Why we can talk about bulging barrels and spinning spirals: Curvature representation in the lexical interface, in E. van der Zee and U. Nikanne (eds), Cognitive Interfaces: Constraints on Linking Cognitive Information, Oxford University Press, Oxford, pp. 143-182.

Zwarts, J.: 2004a, Competition between word meanings: The polysemy of (a)round, in C. Meier and M. Weisgerber (eds), Sinn und Bedeutung, sub8, Proceedings of the eighth meeting of the Gesellschaft für Semantik, Frankfurt, October, 2003, Vol. 114 of University of Konstanz Linguistics Working Papers, Universität Konstanz, Konstanz, pp. 349-360.

Zwarts, J.: 2004b, Prepositional aspect and the algebra of paths. To appear in: Linguistics and Philosophy.

Zwarts, J.: 2006, Event shape: Paths in the semantics of verbs, unpublished manuscript . 